\title{
Existence and stability results of relaxation fractional differential equations with Hilfer-Katugampola fractional derivative
}

\author{
Mohammed. A. Almalahia, ${ }^{a}$, Satish. K. Panchal ${ }^{b}$ \\ ${ }^{a}$ Department of Mathematics, Hajja University, Hajja, Yemen. \\ ${ }^{b}$ Department of Mathematics, Dr. B. A. M. University, Aurangabad, (M.S),431001, India.
}

\begin{abstract}
In this work, we present the existence, uniqueness, and stability result of solution to the nonlinear fractional differential equations involving Hilfer-Katugampola derivative subject to nonlocal fractional integral boundary conditions. The reasoning is mainly based upon properties of Mittag-Leffler functions, and fixed-point methods such as Banach contraction principle and Krasnoselskii's fixed point theorem. Moreover, the generalized Gornwall inequality lemma is used to analyze different types of stability. Finally, one example is given to illustrate our theoretical results.
\end{abstract}

Keywords: Hilfer-Katugampola fractional derivative, Existence of solution, Mittag-Leffler functions, Ulam stability.

$2010 M S C: 26 \mathrm{~A} 33,34 \mathrm{~B} 10,33 \mathrm{E} 12,49 \mathrm{~K} 40$.

\section{Introduction}

Fractional differential equations is very important since their nonlocal property is appropriate to describe memory phenomena such as nonlocal elasticity, propagation in complex medium, biological tissues, polymers, earth sediments, etc, and they have been emerging as an important area of investigation in recent decades. For details, we refer the reader to monographs of Hilfer [10], Kilbas [15], Samko [19], Podlubny [17], and references therein.

Email addresses: aboosama736242107@gmail.com (Mohammed. A. Almalahi), drpanchalsk@gmail.com (Satish. K. Panchal) 
There are various definitions of fractional derivatives, among these definitions, Riemann-Liouville (1832), Riemann (1849), GrunwaldLetnikov (1867), Caputo (1997), Hilfer (2000), as well as Hadamard (1891). At the same context, Kilbas et al. in [15] introduced the properties of fractional integrals and fractional derivatives with respect to another function. O. P. Agrawal et al. in [1, 8], presented the generalized variational calculus in terms of multi-parameters fractional derivatives. Some of generalized fractional integral and differential operators and their properties were introduced by Agrawal in [2]. A Caputo fractional derivative of a function with respect to another function was proposed by R. Almeida in [7]. Recently, Katugampola in [12] introduced a new fractional differential operator. Moreover, this operator has been compounded with Hilfer fractional differential operator introduced by Hilfer [10] which called Hilfer-Katugampola fractional differential operator [16].

Over the last years, the stability results of fractional differential equations have been strongly developed. Very significant contributions about this topic were introduced by Ulam [20, Hyers [11] and this type of stability called Ulam-Hyers stability. The concept of Ulam-Hyers Stability was extended via inserting new function variables provided by Rassias [18] in 1978. Ulam-stability, Ulam-Hyers stability, and Ulam-HyersRassias stability, these labels have become famous today in literature. There are many researchers studied generalized Hilfer fractional differential equations [3, 4, 5, 14].

Recently, Gao et al., in [9] established the existence and uniqueness of solutions to the Hilfer nonlocal boundary value problem

$$
\begin{aligned}
D_{0^{+}}^{p, \beta}(\varsigma)-c y(\varsigma) & =f(\varsigma, y(\varsigma)), c<0,0<p<1,0 \leq \beta \leq 1, \varsigma \in(0, T], \\
I_{0^{+}}^{1-r} y(0) & =\sum_{I=1}^{m} \lambda_{i} I_{0^{+}}^{r} y\left(\tau_{i}\right), p \leq r=p+\beta-p \beta, \tau_{i} \in(0, T],
\end{aligned}
$$

where $D_{0^{+}}^{p, \beta}$ denotes the Hilfer fractional derivative of order $p \in(0,1)$ and type $\beta \in[0,1], I_{0^{+}}^{1-r}$ is the Reimann Liouville fractional integral of order $1-r, r=p+\beta(1-p), c<0$ by using some properties of Hilfer fractional calculus, Mittag-Leffler functions, and fixed point methods. In [6] studied the existence, uniqueness and different types of stabilities of solutions for the following problem:

$$
\begin{aligned}
D_{0^{+}}^{p, \beta} y(\varsigma)-\lambda y(\varsigma) & =f\left(\varsigma, y(\varsigma), D_{0^{+}}^{p, \beta} y(\varsigma)\right), \varsigma \in(0, T] \\
I_{0^{+}}^{1-r} y(0) & =I_{0^{+}}^{1-r} y(T)
\end{aligned}
$$

where ${ }^{H} D_{0^{+}}^{p, \beta}$ denotes the Hilfer fractional derivative of order $p \in(0,1)$ and type $\beta \in[0,1], I_{0^{+}}^{1-r}$ is the Reimann Liouville fractional integral of order $1-r, r=p+\beta(1-p), \lambda<0$.

Motivated by [6, 9], in this paper, we will study Hilfer-Katugampola integral boundary value problems for the following relaxation fractional differential equations:

$$
\left\{\begin{array}{l}
\rho D_{0^{+}}^{\alpha, \beta} y(\varsigma)=\lambda y(\varsigma)+f(\varsigma, y(\varsigma)), \lambda<0,0<\alpha<1,0 \leq \beta \leq 1, \varsigma \in J:=(a, b] \\
{ }^{\rho} I_{0^{+}}^{1-\gamma} y\left(a^{+}\right)=\sum_{i=1}^{m} \delta_{i} \rho I_{0^{+}}^{\eta} y\left(\tau_{i}\right), \alpha \leq \gamma=\alpha+\beta-\alpha \beta<1 \quad \tau_{i} \in(a, b],
\end{array}\right.
$$

where ${ }^{\rho} D_{a^{+}}^{\alpha, \beta}(\cdot)$ denotes the Hilfer-Katugampola fractional derivative of order $\alpha \in(0,1)$ and type $\beta \in[0,1]$ and ${ }^{\rho} I_{0^{+}}^{1-\gamma}$ is a generalized fractional derivative of order $1-\gamma(\gamma=\alpha+\beta-\alpha \beta), \rho>0$. Here $f: J \times \mathbb{R} \longrightarrow \mathbb{R}$ is a given function satisfying some assumptions that will be specified later, $\tau_{i}(i=0,1,2, \ldots, m)$ are prefixed points satisfying $a<\tau_{1} \leq \tau_{2} \leq \ldots . \leq \tau_{m}<b$, and $\lambda<0, \delta_{i} \in \mathbb{R}$.

The paper is organized as follows. In Section 2, we present notations and definitions which are used throughout this paper. In Sect 3, we discuss the existence and uniqueness results for differential equations with Hilfer-Katugampola fractional derivative involving nonlocal initial condition. In Section 4, we discuss different kinds of fractional Ulam stability. 


\section{Preliminaries}

In this section, we present some definitions and lemmas that we will use throughout this paper. Let $0<a<b, J=(a, b]$ and $C[J, \mathbb{R}]$ be the Banach space all continuous functions from $J$ into $\mathbb{R}$ with supremum norm $\|y\|_{\infty}=\sup \{|y(\varsigma)|: \varsigma \in J\}$. For $0<\gamma<1$, we defined the weighted spaces of continuous functions:

$$
C_{\gamma, \rho}[J, \mathbb{R}]=\left\{y:(a, b] \rightarrow \mathbb{R}:\left(\frac{\varsigma^{\rho}-a^{\rho}}{\rho}\right)^{\gamma} y(\varsigma) \in C[J, \mathbb{R}]\right\}
$$

and

$$
C_{\gamma, \rho}^{n}[J, \mathbb{R}]=\left\{y \in C^{n-1}[J, \mathbb{R}]: y^{(n)} \in C_{\gamma, \rho}^{n}[J, \mathbb{R}]\right\}
$$

with the norms

$$
\|y\|_{C_{\gamma, \rho}}=\sup _{\varsigma \in J}\left|\left(\frac{\varsigma^{\rho}-a^{\rho}}{\rho}\right)^{\gamma} f(\varsigma)\right| \text { and }\|y\|_{C_{\gamma, \rho}^{n}}=\sum_{k=0}^{n-1}\left\|y^{(k)}\right\|_{\infty}+\left\|y^{(n)}\right\|_{C_{\gamma, \rho}} .
$$

Conseder the space $X_{c}^{p}(a, b),(c \in \mathbb{R}, 1 \leq p \leq \infty)$ of the complex-valued Lebesgue measurable functions $y$ on $[a, b]$ for which $\|y\|_{X_{c}^{p}}<\infty$, where

$$
\|y\|_{X_{c}^{p}}=\left(\int_{a}^{b}\left|\varsigma^{c} y(\varsigma)\right|^{p} \frac{d \varsigma}{\varsigma}\right)^{\frac{1}{p}} .
$$

In particular, when $c=\frac{1}{p}$, the space $X_{\frac{1}{p}}^{p}(a, b)=L_{p}(a, b)$.

Definition 2.1. [12, 13] Let $\alpha \in \mathbb{R}_{+}, c \in \mathbb{R}$ and $y(\varsigma) \in X_{c}^{p}(a, b)$. The generalized left-sided fractional integral ${ }^{\rho} I_{a^{+}}^{\alpha}$ of order $\alpha>0$ is defined by

$$
{ }^{\rho} I_{a^{+}}^{\alpha} y(\varsigma)=\frac{1}{\Gamma(\alpha)} \int_{a}^{\varsigma}\left(\frac{\varsigma^{\rho}-s^{\rho}}{\rho}\right)^{\alpha-1} s^{\rho-1} y(s) d s, \varsigma>a, \rho>0 .
$$

Definition 2.2. [12, 13] The Katugampola fractional derivative of order $\alpha \in \mathbb{R}_{+} \backslash \mathbb{N}$ and $\rho>0$ is defined by

$$
{ }^{\rho} D_{a^{+}}^{\alpha} y(\varsigma)=\left(\varsigma^{1-\rho} \frac{d}{d \varsigma}\right)^{n} \frac{1}{\Gamma(n-\alpha)} \int_{a}^{b} s^{\rho-1}\left(\frac{\varsigma^{\rho}-s^{\rho}}{\rho}\right)^{n-\alpha-1} y(s) d s,
$$

where $n=[\alpha]+1$.

Definition 2.3. [16] Let $n-1<\alpha<n$ and $0 \leq \beta \leq 1$. The Hilfer-Katugampola fractional derivative with respect to $\varsigma$ with $\rho>0$ of a function $f$ is defined by

$$
{ }^{\rho} D_{a^{+}}^{\alpha, \beta} y(\varsigma)={ }^{\rho} I_{a^{+}}^{\beta(n-\alpha)}\left(\varsigma^{\rho-1} \frac{d}{d \varsigma}\right)^{n}{ }^{\rho} I_{a^{+}}^{(1-\beta)(n-\alpha)} y(\varsigma),
$$

the operator ${ }^{\rho} D_{a^{+}}^{\alpha, \beta}$ can be written as

$$
{ }^{\rho} D_{a^{+}}^{\alpha, \beta}={ }^{\rho} I_{a^{+}}^{\beta(n-\alpha)} \delta_{\rho}^{n}{ }^{\rho} I_{a^{+}}^{n-\gamma}={ }^{\rho} I_{a^{+}}^{\beta(n-\alpha)}{ }^{\rho} D_{a^{+}}^{\gamma}, \quad \gamma=\alpha+n \beta-\alpha \beta,
$$

where

$$
\delta_{\rho}^{n}=\left(\varsigma^{\rho-1} \frac{d}{d \varsigma}\right)^{n}
$$

In this paper we consider $n=1$ because $\alpha \in(0,1)$. 
Lemma 2.1. [16] Let ${ }^{\rho} I_{a^{+}}^{\alpha}$ and ${ }^{\rho} D_{a^{+}}^{\alpha}$ are generalized left-sided fractional integral and derivative which are defined in (2) and (3) respectively. Then for $\varsigma>a$, we have

$$
\begin{aligned}
{\left[{ }^{\rho} I_{a^{+}}^{\alpha}\left(\frac{s^{\alpha}-a^{\rho}}{\rho}\right)^{\beta-1}\right](\varsigma) } & =\frac{\Gamma(\beta)}{\Gamma(\alpha+\beta)}\left(\frac{\varsigma^{\alpha}-a^{\rho}}{\rho}\right)^{\alpha+\beta-1}, \alpha \geq 0, \beta>0 \\
{\left[{ }^{\rho} D_{a^{+}}^{\alpha}\left(\frac{s^{\alpha}-a^{\rho}}{\rho}\right)^{\beta-1}\right](\varsigma) } & =0,0<\alpha<1 .
\end{aligned}
$$

Lemma 2.2. [16] Let $0<\alpha<1,0 \leq \gamma<1$. If $y \in C_{\gamma, \rho}[J, \mathbb{R}]$ and ${ }^{\rho} I_{0^{+}}^{1-\alpha} y(\cdot) \in C_{\gamma, \rho}^{1}[J, \mathbb{R}]$, then for $\varsigma \in J$, we have

$$
{ }^{\rho} I_{a^{+}}^{\alpha}{ }^{\rho} D_{a^{+}}^{\alpha} y(\varsigma)=y(\varsigma)-\frac{{ }^{\rho} I_{a^{+}}^{1-\alpha} y(a)}{\Gamma(\alpha)}\left(\frac{\varsigma^{\alpha}-a^{\rho}}{\rho}\right)^{\alpha-1} .
$$

Lemma 2.3. Let $0<\alpha<1,0 \leq \beta \leq 1$ and $\gamma=\alpha+\beta-\alpha \beta$. If $y \in C_{1-\gamma, \rho}^{\gamma}[J, \mathbb{R}]$, then

$$
{ }^{\rho} I_{a^{+}}^{\gamma}{ }^{\rho} D_{a^{+}}^{\gamma} y(\varsigma)={ }^{\rho} I_{a^{+}}^{\alpha}{ }^{\rho} D_{a^{+}}^{\alpha, \beta} y(\varsigma)
$$

and

$$
{ }^{\rho} D_{a^{+}}^{\gamma}{ }^{\rho} I_{a^{+}}^{\alpha} y(\varsigma)={ }^{\rho} D_{a^{+}}^{\beta(1-\alpha)} y(\varsigma) .
$$

Now, we give definitions of fundamental spaces which we are using to solve our problem. For $\gamma=\alpha+\beta-\alpha \beta$ and $0<\alpha, \beta, \gamma<1$, we define

$$
\begin{aligned}
& C_{1-\gamma, \rho}^{\alpha, \beta}[J, \mathbb{R}]=\left\{y \in C_{1-\gamma, \rho}[J, \mathbb{R}],{ }^{\rho} D_{a^{+}}^{\alpha, \beta} y \in C_{1-\gamma, \rho}[J, \mathbb{R}]\right\} \\
& C_{1-\gamma, \rho}^{\gamma}[J, \mathbb{R}]=\left\{y \in C_{1-\gamma, \rho}[J, \mathbb{R}],{ }^{\rho} D_{a^{+}}^{\gamma} y \in C_{1-\gamma, \rho}[J, \mathbb{R}]\right\}
\end{aligned}
$$

It is clear that $C_{1-\gamma, \rho}^{\gamma}[J, \mathbb{R}] \subset C_{1-\gamma, \rho}^{\alpha, \beta}[J, \mathbb{R}] \subset C_{1-\gamma, \rho}[J, \mathbb{R}]$.

Lemma 2.4. ([22], Lemma 2) Let $\alpha \in(0,2]$ and $\beta>0$ be arbitrary. The function $E_{\alpha}(\cdot), E_{\alpha, \alpha}(\cdot)$ and $E_{\alpha, \beta}(\cdot)$ are nonnegative and for all $z<0$, we have

$$
E_{\alpha}(z):=E_{\alpha, 1}(z) \leq 1, E_{\alpha, \alpha}(z) \leq \frac{1}{\Gamma(\alpha)}, E_{\alpha, \beta}(z) \leq \frac{1}{\Gamma(\beta)} .
$$

Moreover, for any $c<0$ and $\varsigma_{1}, \varsigma_{2} \in[0,1]$,

$$
E_{\alpha, \alpha+\beta}\left(c \varsigma_{2}^{\alpha}\right) \rightarrow E_{\alpha, \alpha+\beta}\left(c \varsigma_{1}^{\alpha}\right) \text { as } \varsigma_{1} \rightarrow \varsigma_{2}
$$

Lemma 2.5. Let $\alpha>0, \beta>0, \gamma>0$ and $\lambda \in \mathbb{R}$. Then

$$
\begin{aligned}
& { }^{\rho} I_{a^{+}}^{\alpha}\left\{\left(\frac{\varsigma^{\rho}-a^{\rho}}{\rho}\right)^{\beta-1} E_{\gamma, \beta}\left[\lambda\left(\frac{\varsigma^{\rho}-a^{\rho}}{\rho}\right)^{\gamma}\right]\right\} \\
& =\left(\frac{\varsigma^{\rho}-a^{\rho}}{\rho}\right)^{\alpha+\beta-1} E_{\gamma, \alpha+\beta}\left[\lambda\left(\frac{\varsigma^{\rho}-a^{\rho}}{\rho}\right)^{\gamma}\right] .
\end{aligned}
$$


Proof. By definition 2.1, we have

$$
\begin{aligned}
& { }^{\rho} I_{a^{+}}^{\alpha}\left(\frac{\varsigma^{\rho}-a^{\rho}}{\rho}\right)^{\beta-1} E_{\gamma, \beta}\left[\lambda\left(\frac{\varsigma^{\rho}-a^{\rho}}{\rho}\right)^{\gamma}\right] \\
& =\frac{1}{\Gamma(\alpha)} \int_{a}^{\varsigma}\left(\frac{\varsigma^{\rho}-s^{\rho}}{\rho}\right)^{\alpha-1} s^{\rho-1}\left(\frac{s^{\rho}-a^{\rho}}{\rho}\right)^{\beta-1} E_{\gamma, \beta}\left[\lambda\left(\frac{s^{\rho}-a^{\rho}}{\rho}\right)^{\gamma}\right] d s \\
& =\frac{1}{\Gamma(\alpha)} \int_{a}^{\varsigma}\left(\frac{\varsigma^{\rho}-s^{\rho}}{\rho}\right)^{\alpha-1} s^{\rho-1}\left(\frac{s^{\rho}-a^{\rho}}{\rho}\right)^{\beta-1} \sum_{n=0}^{\infty} \frac{\left[\lambda\left(\frac{s^{\rho}-a^{\rho}}{\rho}\right)^{\gamma}\right]^{n}}{\Gamma(n \gamma+\beta)} d s \\
& =\sum_{n=0}^{\infty} \frac{\lambda^{n}}{\Gamma(n \gamma+\beta)} \frac{1}{\Gamma(\alpha)} \int_{a}^{\varsigma}\left(\frac{\varsigma^{\rho}-s^{\rho}}{\rho}\right)^{\alpha-1} s^{\rho-1}\left(\frac{s^{\rho}-a^{\rho}}{\rho}\right)^{n \gamma+\beta-1} d s \\
& =\left(\frac{\varsigma^{\rho}-a^{\rho}}{\rho}\right)^{\alpha+\beta-1} \sum_{n=0}^{\infty} \frac{\left[\lambda\left(\frac{\varsigma^{\rho}-a^{\rho}}{\rho}\right)^{\gamma}\right]^{n}}{\Gamma(n \gamma+\alpha+\beta)} \\
& =\left(\frac{\varsigma^{\rho}-a^{\rho}}{\rho}\right)^{\alpha+\beta-1} E_{\gamma, \alpha+\beta}\left[\lambda\left(\frac{\varsigma^{\rho}-a^{\rho}}{\rho}\right)^{\gamma}\right] .
\end{aligned}
$$

Lemma 2.6. Let $\alpha>0, \beta>0, k>0, \lambda \in \mathbb{R}, z \in \mathbb{R}$ and $y \in C[0,1]$. Then

$$
\begin{aligned}
& { }^{\rho} I_{a^{+}}^{k} \int_{0}^{z}\left(\frac{z^{\rho}-\varsigma^{\rho}}{\rho}\right)^{\alpha-1} s^{\rho-1} E_{\alpha, \alpha}\left[\lambda\left(\frac{z^{\rho}-\varsigma^{\rho}}{\rho}\right)^{\alpha}\right] y(\varsigma) d \varsigma \\
& =\int_{0}^{z}\left(\frac{z^{\rho}-\varsigma^{\rho}}{\rho}\right)^{\alpha+k-1} \varsigma^{\rho-1} E_{\alpha, \alpha+k}\left[\lambda\left(\frac{z^{\rho}-\varsigma^{\rho}}{\rho}\right)^{\alpha}\right] y(\varsigma) d \varsigma .
\end{aligned}
$$

Proof. According to definition 2.1 and Lemma 2.5, we obtain

$$
\begin{aligned}
& { }^{\rho} I_{a^{+}}^{k} \int_{a}^{z}\left(\frac{z^{\rho}-\varsigma^{\rho}}{\rho}\right)^{\alpha-1} \varsigma^{\rho-1} E_{\alpha, \alpha}\left[\lambda\left(\frac{z^{\rho}-\varsigma^{\rho}}{\rho}\right)^{\alpha}\right] y(\varsigma) d \varsigma \\
& =\frac{1}{\Gamma(k)} \int_{a}^{z}\left(\frac{z^{\rho}-u^{\rho}}{\rho}\right)^{k-1} u^{\rho-1}\left\{\int_{a}^{u}\left(\frac{u^{\rho}-\varsigma^{\rho}}{\rho}\right)^{\alpha-1} \varsigma^{\rho-1} E_{\alpha, \alpha}\left[\lambda\left(\frac{u^{\rho}-\varsigma^{\rho}}{\rho}\right)^{\alpha}\right] y(\varsigma) d \varsigma\right\} d u \\
& =\frac{1}{\Gamma(k)} \int_{a}^{z} \int_{\varsigma}^{z}\left(\frac{u^{\rho}-\varsigma^{\rho}}{\rho}\right)^{\alpha-1} \varsigma^{\rho-1} E_{\alpha, \alpha}\left[\lambda\left(\frac{u^{\rho}-\varsigma^{\rho}}{\rho}\right)^{\alpha}\right]\left(\frac{z^{\rho}-u^{\rho}}{\rho}\right)^{k-1} u^{\rho-1} y(\varsigma) d u d \varsigma \\
& =\frac{1}{\Gamma(k)} \int_{a}^{z} \varsigma^{\rho-1} y(\varsigma) \int_{\varsigma}^{z}\left(\frac{u^{\rho}-\varsigma^{\rho}}{\rho}\right)^{\alpha-1} E_{\alpha, \alpha}\left[\lambda\left(\frac{u^{\rho}-\varsigma^{\rho}}{\rho}\right)^{\alpha}\right]\left(\frac{z^{\rho}-u^{\rho}}{\rho}\right)^{k-1} u^{\rho-1} d u d \varsigma \\
& =\frac{1}{\Gamma(k)} \int_{a}^{z} y(\varsigma) \Gamma(k)\left(\frac{z^{\rho}-\varsigma^{\rho}}{\rho}\right)^{\alpha+k-1} \varsigma^{\rho-1} E_{\alpha, \alpha+k}\left[\lambda\left(\frac{z^{\rho}-\varsigma^{\rho}}{\rho}\right)^{\alpha}\right] d \varsigma \\
& =\int_{a}^{z}\left(\frac{z^{\rho}-\varsigma^{\rho}}{\rho}\right)^{\alpha+k-1} \varsigma^{\rho-1} E_{\alpha, \alpha+k}\left[\lambda\left(\frac{z^{\rho}-\varsigma^{\rho}}{\rho}\right)^{\alpha}\right] y(\varsigma) d \varsigma .
\end{aligned}
$$

Lemma 2.7. [16] Let $\gamma=\alpha+\beta-\alpha \beta$ where $\alpha \in(0,1), \beta \in[0,1]$ and $g: J \longrightarrow \mathbb{R}$ is a continuous function such that $f \in C_{1-\gamma, \rho}[J, \mathbb{R}]$ for all $y \in C_{1-\gamma, \rho}[J, \mathbb{R}]$. A function $y \in C_{1-\gamma, \rho}^{\gamma}[J, \mathbb{R}]$ is a solution of fractional initial value problem

$$
\left\{\begin{array}{l}
\rho D_{a^{+}}^{\alpha, \beta} y(\varsigma)=\lambda y(\varsigma)+g(\varsigma), \quad \varsigma \in(a, b] \\
\rho I_{a^{+}}^{1-\gamma} y(a)=y_{0}
\end{array}\right.
$$


if and only if $y$ satisfies the following integral

$$
\begin{aligned}
y(\varsigma)= & y_{0}\left(\frac{\varsigma^{\rho}-a^{\rho}}{\rho}\right)^{\gamma-1} E_{\alpha, \gamma}\left[\lambda\left(\frac{\varsigma^{\rho}-a^{\rho}}{\rho}\right)^{\alpha}\right] \\
& +\int_{a}^{\varsigma} s^{\rho-1}\left(\frac{\varsigma^{\rho}-s^{\rho}}{\rho}\right)^{\alpha-1} E_{\alpha, \alpha}\left[\lambda\left(\frac{\varsigma^{\rho}-s^{\rho}}{\rho}\right)^{\alpha}\right] g(s) d s .
\end{aligned}
$$

Proof. see(Hilfer-Katugampola fractional derivatives[16])

Theorem 2.1. Let $\gamma=\alpha+\beta-\alpha \beta$ where $\alpha \in(0,1), \beta \in[0,1]$. If $f: J \times \mathbb{R} \longrightarrow \mathbb{R}$ is a function such that $f(\cdot, y(\cdot)) \in C_{1-\gamma, \rho}[J, \mathbb{R}]$ for all $y \in C_{1-\gamma, \rho}[J, \mathbb{R}]$. A function $y \in C_{1-\gamma, \rho}[J, \mathbb{R}]$ is the solution of problem (1) if and only if $y$ satisfies the following integral equation

$$
y(\varsigma)=\left\{\begin{aligned}
N\left(\frac{\varsigma^{\rho}-a^{\rho}}{\rho}\right)^{\gamma-1} E_{\alpha, \gamma}\left[\lambda\left(\frac{\varsigma^{\rho}-a^{\rho}}{\rho}\right)^{\alpha}\right] \times & \\
& \sum_{i=1}^{m} \delta_{i}{ }^{\rho} I_{a^{+}}^{\eta} \int_{a}^{\tau_{i}} s^{\rho-1}\left(\frac{\tau_{i}^{\rho}-s^{\rho}}{\rho}\right)^{\alpha-1} E_{\alpha, \alpha}\left[\lambda\left(\frac{\tau_{i}^{\rho}-s^{\rho}}{\rho}\right)^{\alpha}\right] f(s, y(s)) d s \\
& +\int_{a}^{\varsigma} s^{\rho-1}\left(\frac{\varsigma^{\rho}-s^{\rho}}{\rho}\right)^{\alpha-1} E_{\alpha, \alpha}\left[\lambda\left(\frac{\varsigma^{\rho}-s^{\rho}}{\rho}\right)^{\alpha}\right] f(s, y(s)) d s,
\end{aligned}\right.
$$

where

$$
N:=\frac{1}{1-\sum_{i=1}^{m} \rho I_{a^{+}}^{\eta} \delta_{i}\left(\frac{\tau_{i}^{\rho}-a^{\rho}}{\rho}\right)^{\gamma-1} E_{\alpha, \gamma}\left[\lambda\left(\frac{\tau_{i}^{\rho}-a^{\rho}}{\rho}\right)^{\alpha}\right]},
$$

and

$$
\sum_{i=1}^{m}{ }^{\rho} I_{a^{+}}^{\eta} \delta_{i}\left(\frac{\tau_{i}^{\rho}-a^{\rho}}{\rho}\right)^{\gamma-1} E_{\alpha, \gamma}\left[\lambda\left(\frac{\tau_{i}^{\rho}-a^{\rho}}{\rho}\right)^{\alpha}\right] \neq 1 .
$$

Proof. Let $y \in C_{1-\gamma, \rho}[J, \mathbb{R}]$ be a solution of the problem (1). Then by lemma 2.7, we have

$$
\begin{aligned}
y(\varsigma)= & { }^{\rho} I_{a^{+}}^{1-\gamma} y\left(a^{+}\right)\left(\frac{\varsigma^{\rho}-a^{\rho}}{\rho}\right)^{\gamma-1} E_{\alpha, \gamma}\left[\lambda\left(\frac{\varsigma^{\rho}-a^{\rho}}{\rho}\right)^{\alpha}\right] \\
& +\int_{a}^{\varsigma} s^{\rho-1}\left(\frac{\varsigma^{\rho}-s^{\rho}}{\rho}\right)^{\alpha-1} E_{\alpha, \alpha}\left[\lambda\left(\frac{\varsigma^{\rho}-s^{\rho}}{\rho}\right)^{\alpha}\right] f(s, y(s)) d s,
\end{aligned}
$$

Next, we substitute $\varsigma=\tau_{i}$ and multiply both side of (6) by $\delta_{i}$ we derive that

$$
\begin{aligned}
\delta_{i} y\left(\tau_{i}\right)= & \delta_{i}{ }^{\rho} I_{a^{+}}^{1-\gamma} y(a)\left(\frac{\tau_{i}^{\rho}-a^{\rho}}{\rho}\right)^{\gamma-1} E_{\alpha, \gamma}\left[\lambda\left(\frac{\tau_{i}^{\rho}-a^{\rho}}{\rho}\right)^{\alpha}\right] \\
& +\delta_{i} \int_{a}^{\tau_{i}} s^{\rho-1}\left(\frac{\tau_{i}^{\rho}-s^{\rho}}{\rho}\right)^{\alpha-1} E_{\alpha, \alpha}\left[\lambda\left(\frac{\tau_{i}^{\rho}-s^{\rho}}{\rho}\right)^{\alpha}\right] f(s, y(s)) d s .
\end{aligned}
$$

Thus, we have

$$
\begin{aligned}
{ }^{\rho} I_{a^{+}}^{1-\gamma} y\left(a^{+}\right)= & \sum_{i=1}^{m} \delta_{i}{ }^{\rho} I_{a^{+}}^{\eta} y\left(\tau_{i}\right)=\sum_{i=1}^{m}{ }^{\rho} I_{a^{+}}^{\eta} \delta_{i} y\left(\tau_{i}\right) \\
= & { }^{\rho} I_{0^{+}}^{1-\gamma} y(a) \sum_{i=1}^{m}{ }^{\rho} I_{a^{+}}^{\eta} \delta_{i}\left(\frac{\tau_{i}^{\rho}-a^{\rho}}{\rho}\right)^{\gamma-1} E_{\alpha, \gamma}\left[\lambda\left(\frac{\tau_{i}^{\rho}-a^{\rho}}{\rho}\right)^{\alpha}\right] \\
& +\sum_{i=1}^{m}{ }^{\rho} I_{a^{+}}^{\eta} \delta_{i} \int_{a}^{\tau_{i}} s^{\rho-1}\left(\frac{\tau_{i}^{\rho}-s^{\rho}}{\rho}\right)^{\alpha-1} E_{\alpha, \alpha}\left[\lambda\left(\frac{\tau_{i}^{\rho}-s^{\rho}}{\rho}\right)^{\alpha}\right] f(s, y(s)) d s .
\end{aligned}
$$

Which implies

$$
{ }^{\rho} I_{a^{+}}^{1-\gamma} y\left(a^{+}\right)=N \sum_{i=1}^{m} \delta_{i}{ }^{\rho} I_{a^{+}}^{\eta} \int_{a}^{\tau_{i}} s^{\rho-1}\left(\frac{\tau_{i}^{\rho}-s^{\rho}}{\rho}\right)^{\alpha-1} E_{\alpha, \alpha}\left[\lambda\left(\frac{\tau_{i}^{\rho}-s^{\rho}}{\rho}\right)^{\alpha}\right] f(s, y(s)) d s,
$$


Substituting (7) into $(6)$ we can derive

$$
\begin{aligned}
y(\varsigma)= & N\left(\frac{\varsigma^{\rho}-a^{\rho}}{\rho}\right)^{\gamma-1} E_{\alpha, \gamma}\left[\lambda\left(\frac{\varsigma^{\rho}-a^{\rho}}{\rho}\right)^{\alpha}\right] \times \\
& \sum_{i=1}^{m} \delta_{i}{ }^{\rho} I_{a^{+}}^{\eta} \int_{a}^{\tau_{i}} s^{\rho-1}\left(\frac{\tau_{i}^{\rho}-s^{\rho}}{\rho}\right)^{\alpha-1} E_{\alpha, \alpha}\left[\lambda\left(\frac{\tau_{i}^{\rho}-s^{\rho}}{\rho}\right)^{\alpha}\right] f(s, y(s)) d s \\
& +\int_{a}^{\varsigma} s^{\rho-1}\left(\frac{\varsigma^{\rho}-s^{\rho}}{\rho}\right)^{\alpha-1} E_{\alpha, \alpha}\left[\lambda\left(\frac{\varsigma^{\rho}-s^{\rho}}{\rho}\right)^{\alpha}\right] f(s, y(s)) d s .
\end{aligned}
$$

Conversely, by the same way of Lemma 2.7 .

\section{Exestence of solution}

Before starting to prove our results, we make the following hypotheses which are needed to prove the existence and unique solutions for our problem.

$\left(H_{1}\right)$ Let $f: J \times \mathbb{R} \rightarrow \mathbb{R}$ be a continuous function and there exist positive constants $L_{f}>0$ such that

$$
|f(\varsigma, u(\varsigma))-f(\varsigma, v(\varsigma))| \leq L_{f}|x-y|
$$

for all $\varsigma \in(0, b], u, v \in \mathbb{R}$.

$\left(H_{2}\right)$ The constant

$$
\Omega=L_{f}\left[\frac{N}{\Gamma(\alpha+\eta+\gamma)} \sum_{i=1}^{m} \delta_{i}\left(\frac{\tau_{i}^{\rho}-a^{\rho}}{\rho}\right)^{\eta+\alpha+\gamma-1}+\frac{\mathcal{B}(\alpha, \gamma)}{\Gamma(\alpha)}\left(\frac{b^{\rho}-a^{\rho}}{\rho}\right)^{\alpha}\right]<1 .
$$

Theorem 3.1. Assume that $\left(H_{1}\right)-\left(H_{2}\right)$ hold. Then the problem (1) has a unique solution in $C_{1-\gamma, \rho}[a, b]$.

Proof. Define the operator $\mathcal{T}_{f}: C_{1-\gamma, \rho}[a, b] \rightarrow C_{1-\gamma, \rho}[a, b]$ by

$$
\mathcal{T}_{f} y(\varsigma)=\left\{\begin{array}{l}
N\left(\frac{\varsigma^{\rho}-a^{\rho}}{\rho}\right)^{\gamma-1} E_{\alpha, \gamma}\left[\lambda\left(\frac{\varsigma^{\rho}-a^{\rho}}{\rho}\right)^{\alpha}\right] \times \\
\sum_{i=1}^{m} \delta_{i}{ }^{\rho} I_{a^{+}}^{\eta} \int_{a}^{\tau_{i}} s^{\rho-1}\left(\frac{\tau_{i}^{\rho}-s^{\rho}}{\rho}\right)^{\alpha-1} E_{\alpha, \alpha}\left[\lambda\left(\frac{\tau_{i}^{\rho}-s^{\rho}}{\rho}\right)^{\alpha}\right] f(s, y(s)) d s \\
\quad+\int_{a}^{\varsigma} s^{\rho-1}\left(\frac{\varsigma^{\rho}-s^{\rho}}{\rho}\right)^{\alpha-1} E_{\alpha, \alpha}\left[\lambda\left(\frac{\varsigma^{\rho}-s^{\rho}}{\rho}\right)^{\alpha}\right] f(s, y(s)) d s .
\end{array}\right.
$$

Note that for any continuous function $f, \mathcal{T}_{f}$ is also continuous. Indeed, for all $\varsigma, \varsigma_{0} \in(a, b]$, we have

$$
\begin{aligned}
& \left|\mathcal{T}_{f} y(\varsigma)-\mathcal{T}_{f} y\left(\varsigma_{0}\right)\right| \\
= & \mid N\left\{\left(\frac{\varsigma^{\rho}-a^{\rho}}{\rho}\right)^{\gamma-1} E_{\alpha, \gamma}\left[\lambda\left(\frac{\varsigma^{\rho}-a^{\rho}}{\rho}\right)^{\alpha}\right]-\left(\frac{\varsigma_{0}^{\rho}-a^{\rho}}{\rho}\right)^{\gamma-1} E_{\alpha, \gamma}\left[\lambda\left(\frac{\varsigma_{0}^{\rho}-a^{\rho}}{\rho}\right)^{\alpha}\right]\right\} \times \\
& \times \sum_{i=1}^{m} \delta_{i}{ }^{\rho} I_{a^{+}}^{\eta} \int_{a}^{\tau_{i}} s^{\rho-1}\left(\frac{\tau_{i}^{\rho}-s^{\rho}}{\rho}\right)^{\alpha-1} E_{\alpha, \alpha}\left[\lambda\left(\frac{\tau_{i}^{\rho}-s^{\rho}}{\rho}\right)^{\alpha}\right] f(s, y(s)) d s \\
& +\int_{a}^{\varsigma} s^{\rho-1}\left(\frac{\varsigma^{\rho}-s^{\rho}}{\rho}\right)^{\alpha-1} E_{\alpha, \alpha}\left[\lambda\left(\frac{\varsigma^{\rho}-s^{\rho}}{\rho}\right)^{\alpha}\right] f(s, y(s)) d s \\
& -\int_{a}^{\varsigma_{0}} s^{\rho-1}\left(\frac{\varsigma_{0}^{\rho}-s^{\rho}}{\rho}\right)^{\alpha-1} E_{\alpha, \alpha}\left[\lambda\left(\frac{\varsigma_{0}^{\rho}-s^{\rho}}{\rho}\right)^{\alpha}\right] f(s, y(s)) d s \mid \\
\rightarrow & 0 \text { as } \varsigma \rightarrow \varsigma_{0}
\end{aligned}
$$


Next, we show that the operator $\mathcal{T}_{f}: C_{1-\gamma, \rho}[a, b] \rightarrow C_{1-\gamma, \rho}[a, b]$ which defined by $10 p$ is a contraction mapping on $C_{1-\gamma, \rho}[a, b]$. By Using Lemmas 2.4 and 2.6 , for $y, v \in C_{1-\gamma, \rho}[J, \mathbb{R}], \varsigma \in(a, b]$, we have

$$
\begin{aligned}
& \left|\left(\frac{\varsigma^{\rho}-a^{\rho}}{\rho}\right)^{1-\gamma}\left[\mathcal{T}_{f} y(\varsigma)-\mathcal{T}_{f} v(\varsigma)\right]\right| \\
& \leq N E_{\alpha, \gamma}\left[\lambda\left(\frac{\varsigma^{\rho}-a^{\rho}}{\rho}\right)^{\alpha}\right] \times \\
& \sum_{i=1}^{m} \delta_{i}{ }^{\rho} I_{a^{+}}^{\eta} \int_{a}^{\tau_{i}} s^{\rho-1}\left(\frac{\tau_{i}^{\rho}-s^{\rho}}{\rho}\right)^{\alpha-1} E_{\alpha, \alpha}\left[\lambda\left(\frac{\tau_{i}^{\rho}-s^{\rho}}{\rho}\right)^{\alpha}\right]|f(s, y(s))-f(s, v(s))| d s \\
& +\left(\frac{\varsigma^{\rho}-a^{\rho}}{\rho}\right)^{1-\gamma} \int_{a}^{\varsigma} s^{\rho-1}\left(\frac{\varsigma^{\rho}-s^{\rho}}{\rho}\right)^{\alpha-1} E_{\alpha, \alpha}\left[\lambda\left(\frac{\varsigma^{\rho}-s^{\rho}}{\rho}\right)^{\alpha}\right]|f(s, y(s))-f(s, v(s))| d s \\
& \leq \frac{N}{\Gamma(\gamma)} \sum_{i=1}^{m} \delta_{i} \int_{a}^{\tau_{i}} s^{\rho-1}\left(\frac{\tau_{i}^{\rho}-s^{\rho}}{\rho}\right)^{\eta+\alpha-1} E_{\alpha, \alpha+\eta}\left[\lambda\left(\frac{\varsigma^{\rho}-s^{\rho}}{\rho}\right)^{\alpha}\right]|f(s, y(s))-f(s, v(s))| d s \\
& +\left(\frac{\varsigma^{\rho}-a^{\rho}}{\rho}\right)^{1-\gamma} \frac{1}{\Gamma(\alpha)} \int_{a}^{\varsigma} s^{\rho-1}\left(\frac{\varsigma^{\rho}-s^{\rho}}{\rho}\right)^{\alpha-1}|f(s, y(s))-f(s, v(s))| d s \\
& \leq \frac{N}{\Gamma(\gamma) \Gamma(\alpha+\eta)} \sum_{i=1}^{m} \delta_{i} \int_{a}^{\tau_{i}} s^{\rho-1}\left(\frac{\tau_{i}^{\rho}-s^{\rho}}{\rho}\right)^{\eta+\alpha-1}|f(s, y(s))-f(s, v(s))| d s \\
& +\left(\frac{\varsigma^{\rho}-a^{\rho}}{\rho}\right)^{1-\gamma} \frac{1}{\Gamma(\alpha)} \int_{a}^{\varsigma} s^{\rho-1}\left(\frac{\varsigma^{\rho}-s^{\rho}}{\rho}\right)^{\alpha-1}|f(s, y(s))-f(s, v(s))| d s \\
& \leq \frac{N L_{f}}{\Gamma(\gamma) \Gamma(\alpha+\eta)} \sum_{i=1}^{m} \delta_{i} \int_{a}^{\tau_{i}} s^{\rho-1}\left(\frac{\tau_{i}^{\rho}-s^{\rho}}{\rho}\right)^{\alpha+\eta-1}|y(s)-v(s)| d s \\
& +\left(\frac{\varsigma^{\rho}-a^{\rho}}{\rho}\right)^{1-\gamma} \frac{L_{f}}{\Gamma(\alpha)} \int_{a}^{\varsigma} s^{\rho-1}\left(\frac{\varsigma^{\rho}-s^{\rho}}{\rho}\right)^{\alpha-1}|y(s)-v(s)| d s \\
& \leq \frac{N L_{f}}{\Gamma(\gamma) \Gamma(\alpha+\eta)} \sum_{i=1}^{m} \delta_{i} \int_{a}^{\tau_{i}} s^{\rho-1}\left(\frac{\tau_{i}^{\rho}-s^{\rho}}{\rho}\right)^{\alpha+\eta-1}\left(\frac{s^{\rho}-a^{\rho}}{\rho}\right)^{\gamma-1}\|y-v\|_{C_{1-\gamma, \rho}[a, b]} d s \\
& +\left(\frac{\varsigma^{\rho}-a^{\rho}}{\rho}\right)^{1-\gamma} \frac{L_{f}}{\Gamma(\alpha)} \int_{a}^{\varsigma} s^{\rho-1}\left(\frac{\varsigma^{\rho}-s^{\rho}}{\rho}\right)^{\alpha-1}\left(\frac{s^{\rho}-a^{\rho}}{\rho}\right)^{\gamma-1}\|y-v\|_{C_{1-\gamma, \rho}[a, b]} d s \\
& \leq L_{f}\left[\frac{N}{\Gamma(\alpha+\eta+\gamma)} \sum_{i=1}^{m} \delta_{i}\left(\frac{\tau_{i}^{\rho}-a^{\rho}}{\rho}\right)^{\eta+\alpha+\gamma-1}+\frac{\mathcal{B}(\alpha, \gamma)}{\Gamma(\alpha)}\left(\frac{\varsigma^{\rho}-a^{\rho}}{\rho}\right)^{\alpha}\right]\|y-v\|_{C_{1-\gamma, \rho}[a, b]},
\end{aligned}
$$

which implies that

$$
\left\|\mathcal{T}_{f} y-\mathcal{T}_{f} v\right\|_{C_{1-\gamma, \rho}} \leq \Omega\|y-v\|_{C_{1-\gamma, \rho}[a, b]} .
$$

Due to $(9)$, the operator $\mathcal{T}$ is a contraction mapping on $C_{1-\gamma, \rho}[a, b]$. According to Banach contraction principle, we deduce that the problem (1) has a unique solution fixed point $y \in C_{1-\gamma, \rho}[a, b]$.

Theorem 3.2. Assume that $\left(H_{1}\right)$ and $\left(H_{2}\right)$ are satisfied. Then the problem (1) has at least one solution in $C_{1-\gamma, \rho}[a, b]$.

Proof. Consider the set $\chi_{r}$ in $C_{1-\gamma, \rho}[a, b]$ defined by

$$
\chi_{r}=\left\{y \in C_{1-\gamma, \rho}[a, b]:\|y\|_{C_{1-\gamma, \rho}[a, b]} \leq r\right\},
$$

with $r \geq \frac{\sigma}{1-\Omega}, \Omega<1$ and

$$
\sigma:=\left[\left(\frac{b^{\rho}-a^{\rho}}{\rho}\right)^{\alpha} \frac{1}{\Gamma(\alpha+1)}+\frac{N}{\Gamma(\alpha+\eta+1)} \sum_{i=1}^{m} \delta_{i}\left(\frac{\tau_{i}^{\rho}-s^{\rho}}{\rho}\right)^{\alpha+\eta}\right] \tilde{f},
$$


where $\tilde{f}:=\sup _{s \in[a, b]}|f(s, 0)|$. Now we subdivide the operator $\mathcal{T}_{f}$ into two operators $A$ and $B$ on $\chi_{r}$ as follows

$$
A y(\varsigma):=\int_{a}^{\varsigma} s^{\rho-1}\left(\frac{\varsigma^{\rho}-s^{\rho}}{\rho}\right)^{\alpha-1} E_{\alpha, \alpha}\left[\lambda\left(\frac{\varsigma^{\rho}-s^{\rho}}{\rho}\right)^{\alpha}\right] f(s, y(s)) d s, \quad \varsigma \in(a, b],
$$

and

$$
\begin{aligned}
B y(\varsigma)= & N\left(\frac{\varsigma^{\rho}-a^{\rho}}{\rho}\right)^{\gamma-1} E_{\alpha, \gamma}\left[\lambda\left(\frac{\varsigma^{\rho}-a^{\rho}}{\rho}\right)^{\alpha}\right] \times \\
& \sum_{i=1}^{m} \delta_{i}{ }^{\rho} I_{a^{+}}^{\eta} \int_{a}^{\tau_{i}} s^{\rho-1}\left(\frac{\tau_{i}^{\rho}-s^{\rho}}{\rho}\right)^{\alpha-1} E_{\alpha, \alpha}\left[\lambda\left(\frac{\tau_{i}^{\rho}-s^{\rho}}{\rho}\right)^{\alpha}\right] f(s, y(s)) d s
\end{aligned}
$$

The proof was divided into several steps as following.

Step (1): We prove that $A y+B z \in \chi_{r}$ for every $y, z \in \chi_{r}$.

i) For operator $A$. According to Lemma 2.4 and for $\varsigma \in(a, b]$, we have

$$
\begin{aligned}
& \left|\left(\frac{\varsigma^{\rho}-a^{\rho}}{\rho}\right)^{1-\gamma} A y(\varsigma)\right| \\
\leq & \left(\frac{\varsigma^{\rho}-a^{\rho}}{\rho}\right)^{1-\gamma} \int_{a}^{\varsigma} s^{\rho-1}\left(\frac{\varsigma^{\rho}-s^{\rho}}{\rho}\right)^{\alpha-1}\left|E_{\alpha, \alpha}\left[\lambda\left(\frac{\varsigma^{\rho}-s^{\rho}}{\rho}\right)^{\alpha}\right] f(s, y(s))\right| d s \\
\leq & \left(\frac{\varsigma^{\rho}-a^{\rho}}{\rho}\right)^{1-\gamma} \frac{1}{\Gamma(\alpha)} \int_{a}^{\varsigma} s^{\rho-1}\left(\frac{\varsigma^{\rho}-s^{\rho}}{\rho}\right)^{\alpha-1}(|f(s, y(s))-f(s, 0)|+|f(s, 0)|) d s \\
\leq & \left(\frac{\varsigma^{\rho}-a^{\rho}}{\rho}\right)^{1-\gamma} \frac{1}{\Gamma(\alpha)} \int_{a}^{\varsigma} s^{\rho-1}\left(\frac{\varsigma^{\rho}-s^{\rho}}{\rho}\right)^{\alpha-1}\left(L_{f}|y(s)|+\widetilde{f}\right) d s \\
\leq & \left(\frac{\varsigma^{\rho}-a^{\rho}}{\rho}\right)^{\alpha} \frac{\mathcal{B}(\alpha, \gamma)}{\Gamma(\alpha)} L_{f}\|y\|_{C_{1-\gamma, \rho}[a, b]}+\left(\frac{\varsigma^{\rho}-a^{\rho}}{\rho}\right)^{\alpha} \frac{\tilde{f}}{\Gamma(\alpha+1)} \\
\leq & \left(\frac{b^{\rho}-a^{\rho}}{\rho}\right)^{\alpha} \frac{\mathcal{B}(\alpha, \gamma)}{\Gamma(\alpha)} L_{f}\|y\|_{C_{1-\gamma, \rho}[a, b]}+\left(\frac{b^{\rho}-a^{\rho}}{\rho}\right)^{\alpha} \frac{\tilde{f}}{\Gamma(\alpha+1)}
\end{aligned}
$$

This gives

$$
\|A y\|_{C_{1-\gamma, \rho}} \leq\left(\frac{b^{\rho}-a^{\rho}}{\rho}\right)^{\alpha} \frac{\mathcal{B}(\alpha, \gamma)}{\Gamma(\alpha)} r L_{f}+\left(\frac{b^{\rho}-a^{\rho}}{\rho}\right)^{\alpha} \frac{\tilde{f}}{\Gamma(\alpha+1)}
$$


ii) For operator $B$. In view of Lemmas 2.4 and 2.6 , we have

$$
\begin{aligned}
& \left|\left(\frac{\varsigma^{\rho}-a^{\rho}}{\rho}\right)^{1-\gamma} B z(\varsigma)\right| \\
= & \mid N E_{\alpha, \gamma}\left[\lambda\left(\frac{\varsigma^{\rho}-a^{\rho}}{\rho}\right)^{\alpha}\right] \sum_{i=1}^{m} \delta_{i} \\
& \rho_{a^{+}}^{\eta} \int_{a}^{\tau_{i}} s^{\rho-1}\left(\frac{\tau_{i}^{\rho}-s^{\rho}}{\rho}\right)^{\alpha-1} E_{\alpha, \alpha}\left[\lambda\left(\frac{\tau_{i}^{\rho}-s^{\rho}}{\rho}\right)^{\alpha}\right] f(s, z(s)) d s \mid \\
\leq & \frac{N}{\Gamma(\gamma)} \sum_{i=1}^{m} \delta_{i} \int_{a}^{\tau_{i}} s^{\rho-1}\left(\frac{\tau_{i}^{\rho}-s^{\rho}}{\rho}\right)^{\alpha+\eta-1}\left|E_{\alpha, \alpha+\eta}\left[\lambda\left(\frac{\tau_{i}^{\rho}-s^{\rho}}{\rho}\right)^{\alpha}\right] f(s, z(s))\right| d s \\
\leq & \frac{N}{\Gamma(\gamma) \Gamma(\alpha+\eta)} \sum_{i=1}^{m} \delta_{i} \int_{a}^{\tau_{i}} s^{\rho-1}\left(\frac{\tau_{i}^{\rho}-s^{\rho}}{\rho}\right)^{\alpha+\eta-1}(|f(s, z(s))-f(s, 0)|+|f(s, 0)|) d s \\
\leq & \frac{N}{\Gamma(\alpha+\eta+\gamma)} \sum_{i=1}^{m} \delta_{i}\left(\frac{\tau_{i}^{\rho}-s^{\rho}}{\rho}\right)^{\alpha+\eta+\gamma-1} L_{f}\|z\|_{C_{1-\gamma, \rho}[a, b]} \\
& +\frac{N}{\Gamma(\alpha+\eta+1)} \sum_{i=1}^{m} \delta_{i}\left(\frac{\tau_{i}^{\rho}-s^{\rho}}{\rho}\right)^{\alpha+\eta} \tilde{f} .
\end{aligned}
$$

Thus we obtain

$$
\begin{aligned}
\|B z\|_{C_{1-\gamma, \rho}} \leq & \frac{N r}{\Gamma(\alpha+\eta+\gamma)} \sum_{i=1}^{m} \delta_{i}\left(\frac{\tau_{i}^{\rho}-s^{\rho}}{\rho}\right)^{\alpha+\eta+\gamma-1} L_{f} \\
& +\frac{N}{\Gamma(\alpha+\eta+1)} \sum_{i=1}^{m} \delta_{i}\left(\frac{\tau_{i}^{\rho}-s^{\rho}}{\rho}\right)^{\alpha+\eta} \tilde{f} .
\end{aligned}
$$

Linking (11) and (12), for every $y, z \in \chi_{r}$, we get

$$
\|A y+B z\|_{C_{1-\gamma, \rho}} \leq\|A y\|_{C_{1-\gamma, \rho}}+\|B z\|_{C_{1-\gamma, \rho}} \leq \Omega r+\sigma \leq r .
$$

Step (2): We prove that $B$ is a contraction mapping. By Theorem 3.1, we have $\mathcal{T}_{f}$ is a contraction mapping on $C_{1-\gamma, \rho}[a, b]$ and hence $B$ is a contraction mapping too.

Step (3): We prove that the operator $A$ is compact and continuous. According to Step 1, we know that

$$
\|A y\|_{C_{1-\gamma, \rho}} \leq\left(\frac{b^{\rho}-a^{\rho}}{\rho}\right)^{\alpha} \frac{\mathcal{B}(\alpha, \gamma)}{\Gamma(\alpha)} r L_{f}+\left(\frac{b^{\rho}-a^{\rho}}{\rho}\right)^{\alpha} \frac{\tilde{f}}{\Gamma(\alpha+1)} .
$$

So the operator $A$ is uniformly bounded. Now we prove the equicontinuous of operator $A$. For any $\varsigma_{1}, \varsigma_{2} \in$ 
$(a, b], \varsigma_{1}<\varsigma_{2}, y \in \chi_{r}$ and using Lemma 2.4 we get

$$
\begin{aligned}
& \left|\left(\frac{\varsigma_{2}^{\rho}-a^{\rho}}{\rho}\right)^{1-\gamma} A y\left(\varsigma_{2}\right)-\left(\frac{\varsigma_{1}^{\rho}-a^{\rho}}{\rho}\right)^{1-\gamma} A y\left(\varsigma_{1}\right)\right| \\
= & \mid\left(\frac{\varsigma_{2}^{\rho}-a^{\rho}}{\rho}\right)^{1-\gamma} \int_{a}^{\varsigma_{2}} s^{\rho-1}\left(\frac{\varsigma_{2}^{\rho}-s^{\rho}}{\rho}\right)^{\alpha-1} E_{\alpha, \alpha}\left[\lambda\left(\frac{\varsigma_{2}^{\rho}-a^{\rho}}{\rho}\right)^{\alpha}\right] \mathcal{F}_{y}(s) d s \\
& -\left(\frac{\varsigma_{1}^{\rho}-a^{\rho}}{\rho}\right)^{1-\gamma} \int_{a}^{\varsigma_{1}} s^{\rho-1}\left(\frac{\varsigma_{1}^{\rho}-s^{\rho}}{\rho}\right)^{\alpha-1} E_{\alpha, \alpha}\left[\lambda\left(\frac{\varsigma_{1}^{\rho}-a^{\rho}}{\rho}\right)^{\alpha}\right] \mathcal{F}_{y}(s) d s \mid \\
\leq & \frac{\left\|\mathcal{F}_{y}(\cdot)\right\|_{C_{1-\gamma, \rho}}}{\Gamma(\alpha)} \mid \int_{a}^{\varsigma_{1}} s^{\rho-1} \\
& \left\{\left(\frac{\varsigma_{2}^{\rho}-s^{\rho}}{\rho}\right)^{\alpha-1}\left(\frac{\varsigma_{2}^{\rho}-a^{\rho}}{\rho}\right)^{1-\gamma}-\left(\frac{\varsigma_{1}^{\rho}-s^{\rho}}{\rho}\right)^{\alpha-1}\left(\frac{\varsigma_{1}^{\rho}-a^{\rho}}{\rho}\right)^{1-\gamma}\right\}\left(\frac{\varsigma^{\rho}-a^{\rho}}{\rho}\right)^{\gamma-1} d s \mid \\
& +\left(\frac{\varsigma_{2}^{\rho}-a^{\rho}}{\rho}\right)^{1-\gamma} \frac{1}{\Gamma(\alpha)}\left|\int_{\varsigma_{1}}^{\varsigma_{2}} s^{\rho-1}\left(\frac{\varsigma_{2}^{\rho}-s^{\rho}}{\rho}\right)^{\alpha-1} \mathcal{F}_{y}(s) d s\right| \\
& \rightarrow 0 \quad \text { as } \varsigma_{2} \rightarrow \varsigma_{1} .
\end{aligned}
$$

Thus $A$ is equicontinuous. By the Arzelà-Ascoli theorem, we deduce that the operator $A$ is compact on $\chi_{r}$. It follows from Krasnoselskii fixed point theorem that the problem (1) has at least one solution in $C_{1-\gamma, \rho}[a, b]$.

\section{Ulam-Hyers and Ulam-Hyers-Rassias stabilities}

In this section, we will discuss the different types of Ulam stability results for Hilfer-Katugampola fractional nonlocal differential equation (1). Let $\epsilon>0$. Consider the problem (1) and below inequality

$$
\left|\rho D_{a^{+}}^{\alpha, \beta} y(\varsigma)-\lambda y(\varsigma)-f(\varsigma, y(\varsigma))\right| \leq \epsilon, \quad \varsigma \in(a, b] .
$$

The following observations are taken from[21, 23, 24]

Lemma 4.1. 24] Let $\alpha>0$ and $x, y$ be two nonnegative function locally integrable on $[a, b]$. Assume that $g$ is nonnegative and nondecreasing. If

$$
x(\varsigma) \leq y(\varsigma)+g(\varsigma) \int_{a}^{\varsigma} s^{\rho-1}\left(\frac{\varsigma^{\rho}-s^{\rho}}{\rho}\right)^{n \alpha-1} x(s) d s, \quad \varsigma \in[a, b] .
$$

Then

$$
x(\varsigma) \leq y(\varsigma)+\int_{a}^{\varsigma} \sum_{n=1}^{\infty} \frac{[g(\varsigma) \Gamma(\alpha)]^{n}}{\Gamma(n \alpha)} s^{\rho-1}\left(\frac{\varsigma^{\rho}-s^{\rho}}{\rho}\right)^{n \alpha-1} y(s) d s, \quad \varsigma \in[a, b] .
$$

If $y$ be a nondecreasing function on $[a, b]$. Then

$$
x(\varsigma) \leq y(\varsigma) E_{\alpha}\left\{g(\varsigma) \Gamma(\alpha)\left(\frac{\varsigma^{\rho}-s^{\rho}}{\rho}\right)^{\alpha}\right\}, \quad \varsigma \in[a, b] .
$$

Definition 4.1. The problem (1) is Ulam-Hyers stable if there exists a real number $\eta_{f}>0$ such that for each $\epsilon>0$ there exists a solution $y \in C_{1-\gamma, \rho}[a, b]$ of the inequality (13) corresponding to a solution $x \in C_{1-\gamma, \rho}[a, b]$ of the problem (1) such that

$$
|y(\varsigma)-x(\varsigma)| \leq \eta_{f} \epsilon, \quad \varsigma \in(a, b] .
$$


Definition 4.2. The problem (1) is generalized Ulam-Hyers stable if there exists $\psi_{f} \in C([0, \infty),[0, \infty))$, $\psi_{f}(0)=0$ such that for each $\eta_{f}>0$ there exists a solution $y \in C_{1-\gamma, \rho}[a, b]$ of the inequality (13) corresponding to a solution $x \in C_{1-\gamma, \rho}[a, b]$ of the problem (1) with

$$
|y(\varsigma)-x(\varsigma)| \leq \psi_{f}(\epsilon), \quad \varsigma \in(a, b] .
$$

Definition 4.3. The problem (1) is Ulam-Hyers-Rassias stable with respect to $\varphi_{\alpha} \in C_{1-\gamma, \rho}[0, b]$ if there exists a real number $\eta_{f, \varphi_{\alpha}}>0$ such that for each $\epsilon>0$ and for each solution $y \in C_{1-\gamma, \rho}[a, b]$ of the inequality

$$
\left|\rho D_{a^{+}}^{\alpha, \beta} y(\varsigma)-\lambda y(\varsigma)-f(\varsigma, y(\varsigma))\right| \leq \epsilon \varphi_{\alpha}(\varsigma), \quad \varsigma \in(a, b],
$$

there exists a solution $x \in C_{1-\gamma, \rho}[a, b]$ of the problem (1) with

$$
|y(\varsigma)-x(\varsigma)| \leq \eta_{f, \varphi_{\alpha}} \epsilon \varphi_{\alpha}(\varsigma), \quad \varsigma \in(a, b] .
$$

Definition 4.4. The problem (1) is generalized Ulam-Hyers-Rassias stable with respect to $\varphi_{\alpha} \in C_{1-\gamma, \rho}[a, b]$ if there exists a real number $\eta_{f, \varphi_{\alpha}}>0$ such that for each $\epsilon>0$ and for each solution $y \in C_{1-\gamma, \rho}^{\gamma}[a, b]$ of the inequality

$$
\left|\rho D_{a^{+}}^{\alpha, \beta} y(\varsigma)-\lambda y(\varsigma)-f(\varsigma, y(\varsigma))\right| \leq \varphi_{\alpha}(\varsigma), \quad \varsigma \in(a, b],
$$

there exists a solution $x \in C_{1-\gamma, \rho}[a, b]$ of the problem (1) with

$$
|y(\varsigma)-x(\varsigma)| \leq \eta_{f, \varphi_{\alpha}} \varphi_{\alpha}(\varsigma), \quad \varsigma \in(a, b] .
$$

Remark 4.1. A function $y \in C_{1-\gamma, \rho}[a, b]$ is a solution of the inequality (13) if and only if there exist a function $z \in C_{1-\gamma, \rho}[a, b]$ such that

(i) $|z(\varsigma)| \leq \epsilon, \quad \varsigma \in(a, b]$;

(ii) ${ }^{\rho} D_{a^{+}}^{\alpha, \beta} y(\varsigma)=\lambda y(\varsigma)+f(\varsigma, y(\varsigma))+z(\varsigma), \varsigma \in(a, b]$.

Lemma 4.2. Let $y \in C_{1-\gamma, \rho}[a, b]$ satisfies the inequality (13). Then y satisfies the following integral inequality

$$
\begin{aligned}
& \left|y(\varsigma)-A_{y}-\int_{a}^{\varsigma} s^{\rho-1}\left(\frac{\varsigma^{\rho}-s^{\rho}}{\rho}\right)^{\alpha-1} E_{\alpha, \alpha}\left[\lambda\left(\frac{\varsigma^{\rho}-s^{\rho}}{\rho}\right)^{\alpha}\right] f(s, y(s)) d s\right| \\
\leq & \left(\frac{N\left(\frac{\varsigma^{\rho}-a^{\rho}}{\rho}\right)^{\gamma-1}}{\Gamma(\gamma) \Gamma(\alpha+\eta+1)} \sum_{i=1}^{m} \delta_{i}\left(\frac{\tau_{i}^{\rho}-s^{\rho}}{\rho}\right)^{\alpha+\eta}+\frac{\left(\frac{b^{\rho}-s^{\rho}}{\rho}\right)^{\alpha}}{\Gamma(\alpha+1)}\right) \epsilon,
\end{aligned}
$$

where

$$
\begin{aligned}
A_{y}= & N\left(\frac{\varsigma^{\rho}-a^{\rho}}{\rho}\right)^{\gamma-1} E_{\alpha, \gamma}\left[\lambda\left(\frac{\varsigma^{\rho}-a^{\rho}}{\rho}\right)^{\alpha}\right] \times \\
& \sum_{i=1}^{m} \delta_{i}{ }^{\rho} I_{a^{+}}^{\eta} \int_{a}^{\tau_{i}} s^{\rho-1}\left(\frac{\tau_{i}^{\rho}-s^{\rho}}{\rho}\right)^{\alpha-1} E_{\alpha, \alpha}\left[\lambda\left(\frac{\tau_{i}^{\rho}-s^{\rho}}{\rho}\right)^{\alpha}\right] f(s, y(s)) d s .
\end{aligned}
$$


Proof. Indeed by Theorem 2.1, and remark 4.1, we have

$$
\begin{aligned}
y(\varsigma)= & N\left(\frac{\varsigma^{\rho}-a^{\rho}}{\rho}\right)^{\gamma-1} E_{\alpha, \gamma}\left[\lambda\left(\frac{\varsigma^{\rho}-a^{\rho}}{\rho}\right)^{\alpha}\right] \times \\
& \sum_{i=1}^{m} \delta_{i}{ }^{\rho} I_{a^{+}}^{\eta} \int_{a}^{\tau_{i}} s^{\rho-1}\left(\frac{\tau_{i}^{\rho}-s^{\rho}}{\rho}\right)^{\alpha-1} E_{\alpha, \alpha}\left[\lambda\left(\frac{\tau_{i}^{\rho}-s^{\rho}}{\rho}\right)^{\alpha}\right] f(s, y(s)) d s \\
& +N\left(\frac{\varsigma^{\rho}-a^{\rho}}{\rho}\right)^{\gamma-1} E_{\alpha, \gamma}\left[\lambda\left(\frac{\varsigma^{\rho}-a^{\rho}}{\rho}\right)^{\alpha}\right] \times \\
& \sum_{i=1}^{m} \delta_{i}{ }^{\rho} I_{a^{+}}^{\eta} \int_{a}^{\tau_{i}} s^{\rho-1}\left(\frac{\tau_{i}^{\rho}-s^{\rho}}{\rho}\right)^{\alpha-1} E_{\alpha, \alpha}\left[\lambda\left(\frac{\tau_{i}^{\rho}-s^{\rho}}{\rho}\right)^{\alpha}\right] z(s) d s \\
& +\int_{a}^{\varsigma} s^{\rho-1}\left(\frac{\varsigma^{\rho}-s^{\rho}}{\rho}\right)^{\alpha-1} E_{\alpha, \alpha}\left[\lambda\left(\frac{\varsigma^{\rho}-s^{\rho}}{\rho}\right)^{\alpha}\right] f(s, y(s)) d s \\
& +\int_{a}^{\varsigma} s^{\rho-1}\left(\frac{\varsigma^{\rho}-s^{\rho}}{\rho}\right)^{\alpha-1} E_{\alpha, \alpha}\left[\lambda\left(\frac{\varsigma^{\rho}-s^{\rho}}{\rho}\right)^{\alpha}\right] z(s) d s .
\end{aligned}
$$

It follows from Lemmas 2.4 and 2.6 , that

$$
\begin{aligned}
\mid y(\varsigma) & -A_{y}-\int_{a}^{\varsigma} s^{\rho-1}\left(\frac{\varsigma^{\rho}-s^{\rho}}{\rho}\right)^{\alpha-1} E_{\alpha, \alpha}\left[\lambda\left(\frac{\varsigma^{\rho}-s^{\rho}}{\rho}\right)^{\alpha}\right] f(s, y(s)) d s \mid \\
= & \mid N\left(\frac{\varsigma^{\rho}-a^{\rho}}{\rho}\right)^{\gamma-1} E_{\alpha, \gamma}\left[\lambda\left(\frac{\varsigma^{\rho}-a^{\rho}}{\rho}\right)^{\alpha}\right] \times \\
& \sum_{i=1}^{m} \delta_{i} \int_{a}^{\tau_{i}} s^{\rho-1}\left(\frac{\tau_{i}^{\rho}-s^{\rho}}{\rho}\right)^{\alpha+\eta-1} E_{\alpha, \alpha+\eta}\left[\lambda\left(\frac{\tau_{i}^{\rho}-s^{\rho}}{\rho}\right)^{\alpha}\right] z(s) d s \\
+ & \int_{a}^{\varsigma} s^{\rho-1}\left(\frac{\varsigma^{\rho}-s^{\rho}}{\rho}\right)^{\alpha-1} E_{\alpha, \alpha}\left[\lambda\left(\frac{\varsigma^{\rho}-s^{\rho}}{\rho}\right)^{\alpha}\right] z(s) d s \mid \\
\leq & \frac{N}{\Gamma(\gamma) \Gamma(\alpha+\eta)}\left(\frac{\varsigma^{\rho}-a^{\rho}}{\rho}\right)^{\gamma-1} \sum_{i=1}^{m} \delta_{i} \int_{a}^{\tau_{i}} s^{\rho-1}\left(\frac{\tau_{i}^{\rho}-s^{\rho}}{\rho}\right)^{\alpha+\eta-1}|z(s)| d s \\
+ & \frac{1}{\Gamma(\alpha)} \int_{a}^{\varsigma} s^{\rho-1}\left(\frac{\varsigma^{\rho}-s^{\rho}}{\rho}\right)^{\alpha-1}|z(s)| d s \\
\leq & \left(\frac{N\left(\frac{\varsigma^{\rho}-a^{\rho}}{\rho}\right)^{\gamma-1}}{\Gamma(\gamma) \Gamma(\alpha+\eta+1)} \sum_{i=1}^{m} \delta_{i}\left(\frac{\tau_{i}^{\rho}-s^{\rho}}{\rho}\right)^{\alpha+\eta}+\frac{\left(\frac{b^{\rho}-s^{\rho}}{\rho}\right)^{\alpha}}{\Gamma(\alpha+1)}\right) \epsilon .
\end{aligned}
$$

Theorem 4.1. Assume that $\left(H_{1}\right)$ and $\left(H_{3}\right)$ are satisfied. Then the problem (1) is Ulam-Hyers stable and generalized Ulam-Hyers stable.

Proof. Let $\epsilon>0$ and $y \in C_{1-\gamma, \rho}[a, b]$ be satisfies the inequality $\left[13\right.$ and $z \in C_{1-\gamma, \rho}[a, b]$ be a unique solution of the nonlocal fractional differential equation

$$
\begin{aligned}
& \rho D_{a^{+}}^{\alpha, \beta} z(\varsigma)=\lambda z(\varsigma)+f(\varsigma, z(\varsigma)), \lambda<0,, \varsigma \in(a, b] \\
& \rho_{I_{a^{+}}^{1-\gamma}} x\left(a^{+}\right)={ }^{\rho} I_{a^{+}}^{1-\gamma} z\left(a^{+}\right),
\end{aligned}
$$

In view of Theorem 2.1, we have

$$
z(\varsigma)=A_{z}+\int_{a}^{\varsigma} s^{\rho-1}\left(\frac{\varsigma^{\rho}-s^{\rho}}{\rho}\right)^{\alpha-1} E_{\alpha, \alpha}\left[\lambda\left(\frac{\varsigma^{\rho}-s^{\rho}}{\rho}\right)^{\alpha}\right] f(s, z(s)) d s,
$$


where

$$
\begin{aligned}
A_{z}= & N\left(\frac{\varsigma^{\rho}-a^{\rho}}{\rho}\right)^{\gamma-1} E_{\alpha, \gamma}\left[\lambda\left(\frac{\varsigma^{\rho}-a^{\rho}}{\rho}\right)^{\alpha}\right] \times \\
& \sum_{i=1}^{m} \delta_{i}{ }^{\rho} I_{a^{+}}^{\eta} \int_{a}^{\tau_{i}} s^{\rho-1}\left(\frac{\tau_{i}^{\rho}-s^{\rho}}{\rho}\right)^{\alpha-1} E_{\alpha, \alpha}\left[\lambda\left(\frac{\tau_{i}^{\rho}-s^{\rho}}{\rho}\right)^{\alpha}\right] f(s, z(s)) d s .
\end{aligned}
$$

By Lemmas 2.4 and 2.6, we can easily show that $A_{z}=A_{y}$. Indeed

$$
\begin{aligned}
\left|A_{z}-A_{y}\right| \leq & N\left(\frac{\varsigma^{\rho}-a^{\rho}}{\rho}\right)^{\gamma-1} E_{\alpha, \gamma}\left[\lambda\left(\frac{\varsigma^{\rho}-a^{\rho}}{\rho}\right)^{\alpha}\right] \times \\
& \sum_{i=1}^{m} \delta_{i}{ }^{\rho} I_{a^{+}}^{\eta} \int_{a}^{\tau_{i}} s^{\rho-1}\left(\frac{\tau_{i}^{\rho}-s^{\rho}}{\rho}\right)^{\alpha-1} \times \\
& E_{\alpha, \alpha}\left[\lambda\left(\frac{\tau_{i}^{\rho}-s^{\rho}}{\rho}\right)^{\alpha}\right]|f(s, z(s))-f(s, y(s))| d s \\
\leq & \frac{N}{\Gamma(\gamma) \Gamma(\alpha+\eta)}\left(\frac{\varsigma^{\rho}-a^{\rho}}{\rho}\right)^{\gamma-1} \sum_{i=1}^{m} \delta_{i} \\
& \int_{a}^{\tau_{i}} s^{\rho-1}\left(\frac{\tau_{i}^{\rho}-s^{\rho}}{\rho}\right)^{\alpha+\eta-1}|f(s, z(s))-f(s, y(s))| d s \\
\leq & \frac{N L_{f}}{\Gamma(\gamma)}\left(\frac{\varsigma^{\rho}-a^{\rho}}{\rho}\right)^{\gamma-1} \sum_{i=1}^{m} \delta_{i}{ }^{\rho} I_{a^{+}}^{\alpha+\eta}|z(s)-y(s)|\left(\tau_{i}\right) \\
= & 0 .
\end{aligned}
$$

Thus

$$
A_{z}=A_{y}
$$

For any $\varsigma \in(0, b]$, we have

$$
\begin{aligned}
|y(\varsigma)-z(\varsigma)| \leq & \left|y(\varsigma)-A_{y}-\int_{a}^{\varsigma} s^{\rho-1}\left(\frac{\varsigma^{\rho}-s^{\rho}}{\rho}\right)^{\alpha-1} E_{\alpha, \alpha}\left[\lambda\left(\frac{\varsigma^{\rho}-s^{\rho}}{\rho}\right)^{\alpha}\right] f(s, y(s)) d s\right| \\
& +\int_{a}^{\varsigma} s^{\rho-1}\left(\frac{\varsigma^{\rho}-s^{\rho}}{\rho}\right)^{\alpha-1} E_{\alpha, \alpha}\left[\lambda\left(\frac{\varsigma^{\rho}-s^{\rho}}{\rho}\right)^{\alpha}\right]|f(s, y(s))-f(s, z(s))| d s \\
\leq & \left|y(\varsigma)-A_{y}-\int_{a}^{\varsigma} s^{\rho-1}\left(\frac{\varsigma^{\rho}-s^{\rho}}{\rho}\right)^{\alpha-1} E_{\alpha, \alpha}\left[\lambda\left(\frac{\varsigma^{\rho}-s^{\rho}}{\rho}\right)^{\alpha}\right] f(s, y(s)) d s\right| \\
& +\frac{L_{f}}{\Gamma(\alpha)} \int_{a}^{\varsigma} s^{\rho-1}\left(\frac{\varsigma^{\rho}-s^{\rho}}{\rho}\right)^{\alpha-1}|y(s)-z(s)| d s \\
\leq & \left(\frac{N\left(\frac{b^{\rho}-a^{\rho}}{\rho}\right)^{\gamma-1}}{\Gamma(\gamma) \Gamma(\alpha+\eta+1)} \sum_{i=1}^{m} \delta_{i}\left(\frac{\tau_{i}^{\rho}-s^{\rho}}{\rho}\right)^{\alpha+\eta}+\frac{\left(\frac{b^{\rho}-s^{\rho}}{\rho}\right)^{\alpha}}{\Gamma(\alpha+1)}\right) \epsilon \\
& +\frac{L_{f}}{\Gamma(\alpha)} \int_{a}^{\varsigma} s^{\rho-1}\left(\frac{\varsigma^{\rho}-s^{\rho}}{\rho}\right)^{\alpha-1}|y(s)-z(s)| d s .
\end{aligned}
$$


By utilizing Lemma 4.1, we get

$$
\begin{aligned}
|x(\varsigma)-y(\varsigma)| & \leq U \epsilon+\int_{a}^{\varsigma}\left(\sum_{n=1}^{\infty} \frac{\left(L_{f}\right)^{n}}{\Gamma(n \alpha)} s^{\rho-1}\left(\frac{\varsigma^{\rho}-s^{\rho}}{\rho}\right)^{n \alpha-1} U \epsilon\right) d s \\
& =U \epsilon\left(1+\sum_{n=1}^{\infty} \frac{\left(L_{f}\right)^{n}}{\Gamma(n \alpha+1)}\left(\frac{\varsigma^{\rho}-a^{\rho}}{\rho}\right)^{n \alpha}\right. \\
& =U \epsilon E_{\alpha}\left(L_{f}\left(\frac{\varsigma^{\rho}-a^{\rho}}{\rho}\right)^{\alpha}\right):=\eta_{f} \epsilon,
\end{aligned}
$$

where $U:=\left(\frac{N\left(\frac{b^{\rho}-a^{\rho}}{\rho}\right)^{\gamma-1}}{\Gamma(\gamma) \Gamma(\alpha+\eta+1)} \sum_{i=1}^{m} \delta_{i}\left(\frac{\tau_{i}^{\rho}-s^{\rho}}{\rho}\right)^{\alpha+\eta}+\frac{\left(\frac{b^{\rho}-s^{\rho}}{\rho}\right)^{\alpha}}{\Gamma(\alpha+1)}\right)$ and $\eta_{f}:=U E_{\alpha}\left(L_{f}\left(\frac{\varsigma^{\rho}-a^{\rho}}{\rho}\right)^{\alpha}\right)$.

Moreover, if we set $\psi(\epsilon)=\eta_{f} \epsilon$, with $\psi(0)=0$ in (15), then problem (1) is generalized Ulam-Hyers stable.

Now, we need to introduce the following hypothesis:

$\left(H_{4}\right)$ There exists an increasing function $\varphi_{\alpha} \in C_{1-\gamma, \rho}[a, b]$ and there exists $\delta_{\varphi_{\alpha}}>0$ such that for any $\varsigma \in(a, b]$

$$
{ }^{\rho} I_{a^{+}}^{\alpha} \varphi_{\alpha}(\varsigma) \leq \delta_{\varphi_{\alpha}} \varphi_{\alpha}(\varsigma)
$$

Theorem 4.2. Assume that $\left(H_{1}\right),\left(H_{3}\right)$ and $\left(H_{4}\right)$ are satisfied. Then, by Definition 4.3 and Definition 4.4 . the problem (1) is Ulam-Hyers-Rassias stable with respect to $\varphi_{\alpha}$ as well as generalized Ulam-Hyers-Rassias stable.

Proof. Let $\epsilon>0$ and $y \in C_{1-\gamma, \rho}[a, b]$ satisfies the inequality

$$
\left|\rho D_{a^{+}}^{\alpha, \beta} y(\varsigma)-\lambda y(\varsigma)-f(\varsigma, y(\varsigma))\right| \leq \epsilon \varphi_{\alpha}(\varsigma), \quad \varsigma \in(a, b] .
$$

Applying ${ }^{\rho} I_{a^{+}}^{\alpha}$ on both sides of the above inequality and using $\left(H_{4}\right)$, we get

$$
\begin{aligned}
& \left|y(\varsigma)-A_{y}-\int_{a}^{\varsigma} s^{\rho-1}\left(\frac{\varsigma^{\rho}-s^{\rho}}{\rho}\right)^{\alpha-1} E_{\alpha, \alpha}\left[\lambda\left(\frac{\varsigma^{\rho}-s^{\rho}}{\rho}\right)^{\alpha}\right] f(s, y(s)) d s\right| \\
\leq & \epsilon \delta_{\varphi_{\alpha}} \varphi_{\alpha}(\varsigma) .
\end{aligned}
$$

Let $x \in C_{1-\gamma, \rho}[a, b]$ be a unique solution of the nonlocal fractional differential equation

$$
{ }^{\rho} D_{0^{+}}^{\alpha, \beta} x(\varsigma)-\lambda x(\varsigma)=f(\varsigma, x(\varsigma)), \quad \varsigma \in(a, b],
$$

with

$$
{ }^{\rho} I_{0^{+}}^{1-\gamma} x\left(a^{+}\right)={ }^{\rho} I_{0^{+}}^{1-\gamma} y\left(a^{+}\right) .
$$

In view of Theorem 2.1, we can derive that

$$
x(\varsigma)=A_{y}+\int_{a}^{\varsigma} s^{\rho-1}\left(\frac{\varsigma^{\rho}-s^{\rho}}{\rho}\right)^{\alpha-1} E_{\alpha, \alpha}\left[\lambda\left(\frac{\varsigma^{\rho}-s^{\rho}}{\rho}\right)^{\alpha}\right] f(s, x(s)) d s,
$$

where

$$
\begin{aligned}
A_{y}= & N\left(\frac{\varsigma^{\rho}-a^{\rho}}{\rho}\right)^{\gamma-1} E_{\alpha, \gamma}\left[\lambda\left(\frac{\varsigma^{\rho}-a^{\rho}}{\rho}\right)^{\alpha}\right] \times \\
& \sum_{i=1}^{m} \delta_{i}{ }^{\rho} I_{a^{+}}^{\eta} \int_{a}^{\tau_{i}} s^{\rho-1}\left(\frac{\tau_{i}^{\rho}-s^{\rho}}{\rho}\right)^{\alpha-1} E_{\alpha, \alpha}\left[\lambda\left(\frac{\tau_{i}^{\rho}-s^{\rho}}{\rho}\right)^{\alpha}\right] f(s, y(s)) d s .
\end{aligned}
$$


On the other hand, by utilizing (17) and Lemma 2.4, we can get

$$
\begin{aligned}
|y(\varsigma)-x(\varsigma)| \leq & \left|y(\varsigma)-A_{y}-\int_{a}^{\varsigma} s^{\rho-1}\left(\frac{\varsigma^{\rho}-s^{\rho}}{\rho}\right)^{\alpha-1} E_{\alpha, \alpha}\left[\lambda\left(\frac{\varsigma^{\rho}-s^{\rho}}{\rho}\right)^{\alpha}\right] f(s, y(s)) d s\right| \\
& +\int_{a}^{\varsigma} s^{\rho-1}\left(\frac{\varsigma^{\rho}-s^{\rho}}{\rho}\right)^{\alpha-1} E_{\alpha, \alpha}\left[\lambda\left(\frac{\varsigma^{\rho}-s^{\rho}}{\rho}\right)^{\alpha}\right]|f(s, y(s))-f(s, x(s))| d s \\
\leq & \epsilon \delta_{\varphi_{\alpha}} \varphi_{\alpha}(\varsigma)+\frac{L_{f}}{\Gamma(\alpha)} \int_{a}^{\varsigma} s^{\rho-1}\left(\frac{\varsigma^{\rho}-s^{\rho}}{\rho}\right)^{\alpha-1}|y(s)-x(s)| d s .
\end{aligned}
$$

Apply Lemma 4.1, we derive

$$
\begin{aligned}
& |y(\varsigma)-x(\varsigma)| \\
\leq & \epsilon \delta_{\varphi_{\alpha}} \varphi_{\alpha}(\varsigma)+\int_{0}^{\varsigma}\left(\sum_{n=1}^{\infty} \frac{\left(L_{f}\right)^{n}}{\Gamma(n \alpha)} s^{\rho-1}\left(\frac{\varsigma^{\rho}-s^{\rho}}{\rho}\right)^{n \alpha-1} \epsilon \delta_{\varphi_{\alpha}} \varphi_{\alpha}(s)\right) d s \\
\leq & \epsilon \delta_{\varphi_{\alpha}} \varphi_{\alpha}(\varsigma)+\epsilon \delta_{\varphi_{\alpha}} \int_{0}^{\varsigma}\left(\sum_{n=1}^{\infty} \frac{\left(L_{f}\right)^{n}}{\Gamma(n \alpha)} s^{\rho-1}\left(\frac{\varsigma^{\rho}-s^{\rho}}{\rho}\right)^{n \alpha-1} \varphi_{\alpha}(s)\right) d s \\
\leq & \epsilon \delta_{\varphi_{\alpha}} \varphi_{\alpha}(\varsigma)+\epsilon \delta_{\varphi_{\alpha}} \sum_{n=1}^{\infty}\left(L_{f} \delta_{\varphi_{\alpha}}\right)^{n} \varphi_{\alpha}(\varsigma) \\
\leq & \left(1+\sum_{n=1}^{\infty}\left(L_{f} \delta_{\varphi_{\alpha}}\right)^{n}\right) \epsilon \delta_{\varphi_{\alpha}} \varphi_{\alpha}(\varsigma) \\
= & \epsilon\left(1+\sum_{n=1}^{\infty}\left(L_{f} \delta_{\varphi_{\alpha}}\right)^{n}\right) \delta_{\varphi_{\alpha}} \varphi_{\alpha}(\varsigma)=\eta_{f, \varphi_{\alpha}} \epsilon \varphi_{\alpha}(\varsigma),
\end{aligned}
$$

where $\eta_{f, \varphi_{\alpha}}:=\left(1+\sum_{n=1}^{\infty}\left(L_{f} \delta_{\varphi_{\alpha}}\right)^{n}\right) \delta_{\varphi_{\alpha}}$. So

$$
|x(\varsigma)-y(\varsigma)| \leq \eta_{f, \varphi_{\alpha}} \epsilon \varphi_{\alpha}(\varsigma) .
$$

Thus, the problem (1) is Ulam-Hyers Rassias stable. Moreover, an argument similar to above in the previous steps with putting $\epsilon=1$, we get

$$
|x(\varsigma)-y(\varsigma)| \leq \eta_{f, \varphi_{\alpha}} \varphi_{\alpha}(\varsigma) .
$$

This proves that the problem (1) is generalized Ulam-Hyers Rassias stable.

\section{An example}

In this section, one example is given to illustrate our theory results

Example 5.1. Consider the following problem

$$
\left\{\begin{array}{l}
{ }^{1} D_{0^{+}}^{\frac{1}{2}, \frac{2}{3}} y(\varsigma)=-\frac{1}{2} y(\varsigma)+\frac{e^{\varsigma}}{1+e^{\varsigma}} \sin y(\varsigma), \quad \varsigma \in J:=(0,1], \\
{ }^{1} I_{0^{+}}^{\frac{1}{6}} y(0)=\frac{2}{3} I_{0^{+}}^{\frac{1}{2}} y\left(\frac{1}{2}\right) .
\end{array}\right.
$$

Here $\alpha=\frac{1}{2}, \beta=\frac{2}{3}, \gamma=\alpha+\beta-\alpha \beta=\frac{5}{6}, m=1, \tau_{1}=\frac{1}{2}, \delta_{1}=\frac{2}{3}, \eta=\frac{1}{2},(a, b]=(0,1], \rho=1, \lambda=-\frac{1}{2}$ and $f(\varsigma, y(\varsigma))=\frac{e^{\varsigma}}{1+e^{\varsigma}} \sin y(\varsigma)$. Then

$$
\begin{aligned}
|f(\varsigma, z(\varsigma))-f(\varsigma, y(\varsigma))| & \leq\left|\frac{e^{\varsigma}}{1+e^{\varsigma}}(\sin z(\varsigma)-\sin y(\varsigma))\right| \\
& \leq \frac{e}{2}|z(\varsigma)-y(\varsigma)| .
\end{aligned}
$$

We note that $L_{f}=\frac{e}{2}$. Furthermore, by simple calculation, we get $\Omega \simeq 0.67<1$. Then all the assumptions in Theorem 3.1 are satisfied, the problem (20) has a unique solution in $C_{\frac{1}{6}, 1}[0,1]$. 


\section{Conclusion}

Here the existence, uniqueness and stability of nonlocal boundary value problem for differential equation with Hilfer-Katugampola fractional derivative is discussed. Krasnoselskii fixed point theorem, Banach contraction principle, and Ulam type stability are utilized to obtain results. In conclusion, Hilfer-Katugampola fractional derivative can be used as a powerful tool for studying the dynamical behavior of many real-world problems.

\section{Acknowledgments}

The authors thank the referees for their careful reading of the manuscript and insightful comments, which helped to improve the quality of the paper. We would also like to acknowledge the valuable comments and suggestions from the editors, which vastly contributed to improving the presentation of the paper.

\section{References}

[1] O.P. Agrawal, S.I. Muslih, D. Baleanu, Generalized variational calculus in terms of multi-parameters fractional derivatives, Communications in Nonlinear Science and Numerical Simulation. 16(12) (2011) 4756-4767.

[2] O.P. Agrawal, Some generalized fractional calculus operators and their applications in integral equations, Fract. Calc. Appl. Anal. 15 (2012) 700-711.

[3] M.A. Almalahi, M.S. Abdo, S.K. Panchal, $\psi$-Hilfer Fractional functional differential equation by Picard operator method. Journal of Nonlinear Dynamics (2020)

[4] M.A. Almalahi, S.K. Panchal, $\mathrm{E}_{\alpha}$-Ulam-Hyers stability result for $\psi$-Hilfer Nonlocal Fractional Differential Equation. Discontinuity, Nonlinearity, and Complexity (2020)

[5] M.A. Almalahi, M.S. Abdo, S.K. Panchal, Existence and Ulam-Hyers-Mittag-Leffler stability results of $\psi$-Hilfer nonlocal Cauchy problem. Rend. Circ. Mat. Palermo, II. Ser (2020). https://doi.org/10.1007/s12215-020-00484-8

[6] M.A. Almalahi, M.S. Abdo, S.K. Panchal, Periodic boundary value problems for fractional implicit differential equations involving Hilfer fractional derivative. 9(2) (2020).

[7] R. Almeida, A Caputo fractional derivative of a function with respect to another function, Commun. Nonlinear Sci. Numer. Simul., 44 (2017) 460-481.

[8] D. Baleanu, O.P. Agrawal, S. I. Muslih, Lagrangians with linear velocities within Hilfer fractional derivative. In ASME 2011 International Design Engineering Technical Conferences and Computers and Information in Engineering Conference, American Society of Mechanical Engineers Digital Collection, (2011) 335-338).

[9] Z. Gao, Yu, X, Existence results for BVP of a class of Hilfer fractional differential equations. Journal of Applied Mathematics and Computing, 56(1-2) (2018) 217-233.

[10] R. Hilfer, Applications of Fractional Calculus in Physics, World scienti c, Singapore, 1999.

[11] D.H. Hyers, G. Isac, Th.M. Rassias, Stability of Functional Equations in Several Variables, Progr. Nonlinear Differential Equations Appl., Birkh 646user, Boston, 34 (1998).

[12] U.N. Katugampola, New approach to a genaralized fractional integral, Appl. Math.Comput., 218(2011), no. 3, 860-865.

[13] U.N. Katugampola, Existence and uniqueness results for a class of generalized fractional differential equations, Bull. Math. Anal. Appl., 1(2014).

[14] U.N. Katugampola, New fractional integral unifying six existing fractional integrals, epint arxiv: $1612.08596,6$ pages. (2016).

[15] A.A. Kilbas, H. M. Srivastava, J. J. Trujillo, Theory and Applications of Fractional Differential Equations. North-Holland Mathematics Studies, Elsevier, Amsterdam, 207 (2006).

[16] D.S. Oliveira, de oliveira E. Capelas, Hilfer-Katugampola fractional derivative. Comp Appl Math, (2017), 37: 3672-3690

[17] I. Podlubny, Fractional Differential Equations: An Introduction to Fractional Derivatives, Fractional Differential Equations, to Methods of Their Solution and Some of Their Applications, Math. Sci. Eng. 198, Elsevier, Amsterdam, 1999.

[18] T.M. Rassias, On the stability of the linear mapping in Banach spaces, Proc. Amer. Math. Soc., 72(2) (1978), $297-300$.

[19] S.G. Samko, A.A. Kilbas, O.I. Marichev, Fractional Integrals and Derivatives: Theory and Applications, Gordon and Breach, Yverdon (1987).

[20] S.M. Ulam, A Collection of Mathematical Problems, Interscience Tracts in Pure and Applied Mathematics, 8, Inter-science, New York-London(1960).

[21] da C Sousa J. Vanterler, de Oliveira E. Capelas, Ulam-Hyers stability of a nonlinear fractional Volterra integro-differential equation. Appl Math Lett, (2018), 81: 50-56.

[22] J.R. Wang, M. Feckan, Y. Zhou, Presentation of solutions of impulsive fractional Langevin equations and existence results. Eur. Phys. J. Spec. Top. 222 (2013) 1857-1874.

[23] J.R. Wang, L Lv, Y Zhou, Ulam stability and data dependence for fractional differential equations with Caputo derivative. Electron J Qual Theory Differ Equ, (2011), 63: 1-10.

[24] H. Ye, J. Gao, Y. Ding, A generalized Gronwall inequality and its application to a fractional differential equation. J Math Anal Appl, (2007), 328: 1075-1081. 